\title{
Planificación urbana e innovación: de la tecnología y la práctica profesional a la innovación social y política*
}

\author{
Antònia Casellas \\ Universitat Autònoma de Barcelona \\ antonia.casellas@uab.cat \\ Corrado Poli \\ Università di Comunicazione e Lingue (IULM), Milano \\ policorrado@gmail.com
}

Recepción: diciembre de 2012

Aceptación: junio de 2013

\section{Resumen}

La contaminación y los problemas del medio ambiente, delitos menores, la congestión del tráfico, la democracia y la justicia social son preocupaciones constantes en grandes ciudades contemporáneas y áreas metropolitanas. Este artículo argumenta la necesidad de innovar en la política y la gestión urbana para hacer frente a estos problemas con eficacia. La innovación técnica, política y cultural implica un análisis crítico de la influencia de las organizaciones sociales - incluyendo los colegios profesionales - en los procesos de toma de decisiones de los gobiernos y en la asignación de la inversión. La crisis económica actual hace que un cambio significativo en la rutina cotidiana sea más factible que en el pasado. La introducción de nuevas tecnologías implica un replanteamiento de las prioridades en obras públicas y construcción de infraestructura. Un cambio profundo en la geografía urbana dependerá de una revolución cultural y tecnológica; por ello, este análisis aborda temas como: la dinámica de poder, la percepción sobre la innovación efectiva por parte de votantes y agentes públicos y las políticas de investigación y desarrollo. El artículo desarrolla las implicaciones de este enfoque en el ámbito de la movilidad.

Palabras clave: innovación; planificación urbana; tecnología; movilidad.

* Este trabajo se inscribe en la investigación financiada con una ayuda para acciones de cooperación en el marco de la Comunitat de Treball dels Pirineus AC065723 (2010 XI-CTP) de la Agència de Gestió d'Ajuts Universitaris i de Recerca de la Generalitat de Catalunya; la Acción Complementaria (CS 02011-13651-E) del Ministerio de Ciencia e Innovación, y el subprograma Ramón y Cajal del Ministerio de Ciencia e Innovación (RYC-2008-02456). 
Resum. Planificació urbana i innovació: de la tecnologia i la pràctica professional a la innovació social i politica

La contaminació i els problemes del medi ambient, delictes menors, la congestió del trànsit, la democràcia i la justícia social són preocupacions constants en grans ciutats contemporànies i àrees metropolitanes. Aquest article argumenta la necessitat d'innovar en la política i la gestió urbana per fer front a aquests problemes amb eficàcia. La innovació tècnica, política i cultural inclou una anàlisi crítica de la influència de les organitzacions socials -incloent-hi els col-legis professionals - en els processos de presa de decisions dels governs i en l'assignació de la inversió. La crisi econòmica actual fa que un canvi significatiu en la rutina quotidiana sigui més factible que en el passat. La introducció de noves tecnologies implica un replantejament de les prioritats en obres públiques i construcció d'infraestructura. Un canvi profund en la geografia urbana dependrà d'una revolució cultural i tecnològica; per això, aquesta anàlisi aborda temes com ara: la dinàmica de poder, la percepció sobre la innovació efectiva per part de votants i agents públics i les polítiques de recerca i desenvolupament. L'article desenvolupa les implicacions d'aquest enfocament en l'àmbit de la mobilitat.

Paraules clau: innovació; planificació urbana; tecnologia; mobilitat.

Résumé. Aménagement urbain et innovation: de la technologie et de l'organisation professionnelle à l'innovation sociale et politique

Les problèmes environnementaux, la petite délinquance, la congestion du trafic, la démocratie locale et la justice social sont, aujourd'hui, les préoccupations principaux pour les gouvernements des villes et des les agglomérations métropolitaines. Pour faire face efficacement à ces problèmes, il est nécessaire innover l'aménagement urbaine sous le point de vue soit politique, soit technique. Pour favoriser l'innovation technique, politique et culturale il faut élaborer une analyse critique de l'influence des organisations sociales - y compris les corporations professionnelles - dans les processus de décision et dans la répartition des investissements publics. La crise économique rend possible des changements profonds mieux que dans le passé. L'introduction des nouvelles technologies implique un remaniement des priorités en ce qui concerne les travaux publics et la construction des infrastructures. Une transformation radicale de la géographie urbaine pourrait être provoqué par une révolution culturelle et technologique, donc cette analyse est concentrée sur thèmes tels que : les dynamiques du pouvoir, la perception de l'innovation efficace par les électeurs et les décideurs, et les stratégies de la recherche et développement. Cet essai propose aussi un cas d'étude sur la mobilité urbaine.

Mots clé: innovation; aménagement urbain; technologie; mobilité.

\section{Abstract. Urban planning and innovation: from technology and professional practice to social and political innovation}

Pollution and environmental issues, petty crime, traffic congestion, democracy and social justice are major concerns in contemporary larger cities and metropolitan areas. This paper calls for innovation in urban policy and management to deal with these problems effectively. Technical, political and cultural innovation includes a critical analysis of the influence of social organizations - including professional guilds — in government decision-making processes and in investment allocation. The current economic crisis makes some dramatic change in the ordinary routine more possible than in the past. The introduction of new technologies implies a re-thinking of priorities in public works and infrastructure build- 
ing. A possible thorough change in urban geography will be a consequence of a cultural and technological revolution, for this reason this analysis focuses on issues such as: power dynamics; perception of effective innovation by voters and decision-makers; and research and development policies. The paper develops the implications of this approach in the area of mobility.

Keywords: innovation; urban planning; technology; mobility.

\section{Sumario}

Introducción

Tecnología, innovación y sociedad: una reflexión de contexto

Las dinámicas reactivas en la organización

del espacio urbano
La movilidad a partir de la innovación política y social

Reflexiones finales

Bibliografía

\section{Introducción}

A pesar de las extraordinarias inversiones tanto públicas como privadas realizadas en las décadas previas a la presente crisis, las grandes ciudades y áreas metropolitanas se encuentran en una situación crítica con respecto a cuestiones tales como el medio ambiente, la congestión del tráfico, la democracia y la justicia social. Sumándose a esta problemática, en respuesta a la crisis iniciada en el 2007, las intensas y crecientes restricciones presupuestarias han limitado considerablemente el gasto público y han introducido nuevas deficiencias en la provisión de servicios urbanos y en la mejora de las externalidades negativas generadas por el urbanismo expansivo de los últimos años. A estas limitaciones cabe añadir la falta de inversión privada, que en el caso de España se ve acuciada por un alto nivel de endeudamiento, tanto de empresas como de familias. Frente a este contexto, el presente artículo argumenta que, si bien las dinámicas territoriales existentes ilustran el fracaso del pasado enfoque, la crisis actual crea la oportunidad y amplifica la necesidad de repensar la posibilidad de nuevas dinámicas territoriales, resultantes de una comprensión diferente de la innovación y la planificación.

Para desarrollar este argumento, el artículo avanza la idea de que la innovación debe ser entendida en primer lugar como innovación política y social. Esta perspectiva tiene como fin revertir la percepción dominante sobre la innovación, la cual hasta ahora se ha definido principalmente desde un punto de vista técnico, dominado por grupos de presión autorreferenciales, tanto de carácter profesional como económico. En esta línea, se argumenta que el concepto de innovación entendida meramente como aspecto técnico ha permitido tan solo una adaptabilidad de las políticas y estrategias de regeneración urbana de forma reactiva, es decir, desarrollando y ejecutando mecanismos que respondían a intereses y necesidades económicas en lugar de crear e investigar alternativas 
nuevas. El documento desarrolla las consecuencias que este enfoque tiene en políticas urbanas y propone una nueva visión a través del análisis de la movilidad.

En la siguiente sección se presenta una reflexión acerca de la relación entre tecnología, innovación y sociedad, y su influencia en las dinámicas territoriales. Desde la perspectiva epistemológica se cuestiona las posibles interpretaciones acerca de los conceptos de nueva tecnología y dinámica urbana, apuntando a los riesgos de una sociedad entendida como pura tecnología social. A continuación se argumenta que, a pesar del uso y el potencial de las nuevas tecnologías, la ciudad contemporánea no se ha modificado a partir de las potencialidades que ofrecen las nuevas tecnologías de la comunicación y la información. Para articular este argumento, en este apartado se identifican las características relevantes del sistema capitalista antes de la crisis y se exponen las directrices que se han ejecutado en políticas públicas en respuesta a los imperativos económicos que han operado como motor de las políticas urbanas. En la última sección, aplicando el concepto de innovación desde el ámbito político y social, se analiza el caso de la movilidad. Este caso se presenta como ejemplo del potencial de un nuevo enfoque que, desde una nueva comprensión y aplicación de la innovación entendida como proceso social y político, permite avanzar en la aplicabilidad del potencial tecnológico con el fin de generar un nuevo espacio urbano que responda de forma más flexible a las necesidades de una sociedad plural y en proceso de cambio.

\section{Tecnología, innovación y sociedad: una reflexión de contexto}

Una de las preguntas fundamentales para afrontar el tema de la innovación y la planificación territorial hace referencia a la cuestión de cómo las nuevas tecnologías influyen en las dinámicas territoriales. Para responder a esta pregunta se hace necesario abordar de forma específica qué entendemos por nuevas tecnologías y por dinámicas urbanas.

Con respecto a las tecnologías, se debe reflexionar sobre dos tipos de interrogantes. En primer lugar, cabe preguntarse sobre los contenidos de la tecnología, es decir, debemos cuestionarnos acerca de qué tecnologías específicas se deben utilizar para facilitar el desarrollo territorial, tomando en consideración aspectos no tan solo económicos, sino también de equilibrio social y medioambiental. En segundo lugar, y de forma mucho más relevante, hay que tener en cuenta una secuencia fundamental que incluye un análisis a las preguntas de ¿quién inventa, quién produce y quién aplica las nuevas potencialidades tecnológicas? En este marco, debemos considerar el potencial de las nuevas tecnologías que todavía no han sido introducidas en los procesos de desarrollo territorial; y, aún más importante, comprender las razones por las cuales se siguen aplicando tecnologías y estrategias tradicionales — por ejemplo, grandes inversiones en infraestructura para la movilidad-, aunque desde hace tiempo se ha demostrado que no son capaces de solucionar los problemas del tráfico. 
Con respecto a las dinámicas urbanas, y abordando la primera pregunta, podemos cuestionarnos si la innovación es el producto de tecnologías que se eligen y elaboran libremente, o si las tecnologías adoptadas son la respuesta que los técnicos dan a una evolución urbana que se produce autónomamente, fuera de nuestro control. Inicialmente, el concepto de nuevas tecnologías nos remite a un objeto o proceso elaborado por técnicos o ingenieros, a menudo sin una reflexión o control social y político previo. Es decir, en general, cuando se piensa en tecnología no se tiene en cuenta la secuencia anteriormente mencionada sobre quién inventa, quién produce y quién aplica. En este caso, la tecnología - ya sea nueva o vieja — se superpone a la sociedad, que se asume como un dato o una constante fija.

Sin embargo, cabe enfatizar que a menudo la sociedad acaba por convertirse en una variable dependiente de las tecnologías. Es decir, una vez que el proceso se ha iniciado, el progreso técnico incide de manera profunda en el modo en que la sociedad se organiza. Si tenemos fe en la técnica y en la tecnocracia, la capacidad de la tecnología de moldear nuestra sociedad puede interpretarse como una noticia positiva. De hecho, quizá durante un cierto tiempo así fue. Sin embargo, desde hace unas décadas parece claro que han surgido notables problemas y dudas sobre la sociedad del riesgo tecnológico. Como argumenta Ulrick Beck (1992), el creciente riesgo global es la consecuencia de una fase de desarrollo de la sociedad moderna en la que los factores de riesgos sociales, políticos, ecológicos e individuales son creados por el impulso de una innovación que elude de forma creciente el control de las instituciones de la sociedad industrial tradicional.

En la dialéctica entre tecnología y sociedad podemos argumentar que el problema aparece cuando se pierde el control sobre la tecnología, de tal modo que no logramos manejarnos ni organizarnos de acuerdo con principios, valores y acciones humanas y políticas elegidas de forma libre y racionalmente. Los principios de libertad y de racionalidad nacieron con la modernidad y con la Ilustración, y se sintetizaron con el lema kantiano de sapere aude. Sin embargo, si perdemos el control de la acción humana, la sociedad misma se transforma en tecnología social — como la ha definido Niklas Luhmann (1990) y sus seguidores-, es decir, en un sistema en el que los actores no tienen la capacidad de intervenir. En este caso, la tecnología avanza de forma autónoma y tiene la característica de la autorreferencialidad. Podemos por ello argumentar que es posible ver en la aplicación tecnológica una cierta peligrosidad.

La cuestión de la autorreferencialidad de la tecnología es un problema que no se puede obviar, aunque a corto plazo no sea fácil. En la práctica cotidiana la sociedad reducida a pura tecnología social comporta la exclusión de un análisis crítico sobre lo que es adecuado y lo que no lo es. Pero, y de forma aún más importante, no permite definir las finalidades de la acción y de la tecnología. En este tipo de situaciones, los agentes involucrados en el sistema social no consiguen ejercitar una autoridad sobre la tecnología a través de la acción política y democrática. Es decir, por medio de la elaboración de un pensamiento autónomo respecto a la técnica y, por lo tanto, realmente creativo. 


\section{Las dinámicas reactivas en la organización del espacio urbano}

La ciudad contemporánea todavía no ha empezado a modificarse a partir de la disponibilidad y el uso común de las nuevas tecnologías de la comunicación. Cada día utilizamos internet y móviles para servicios tan variados que nos sorprende la versatilidad de las nuevas tecnologías. De hecho, consideramos que la telemática y las telecomunicaciones son una parte importante de nuestra vida y que con su incorporación hemos cambiado profundamente nuestras costumbres. Ello es cierto solo en parte. La conformación de las ciudades y de gran parte de organizaciones materiales y burocráticas no ha cambiado substancialmente. Y tampoco ha cambiado nuestra mentalidad.

La reestructuración económica a través de diferentes periodos de crecimiento y crisis ha condicionado, en las últimas décadas, la organización del espacio urbano y las políticas públicas. Sin embargo, estas políticas se han adaptado en respuesta a los nuevos retos, a menudo al margen de la aplicación innovadora de nuevos modelos sociales y políticos. Una posible línea de actuación innovadora podría haberse orientado hacia la renaturalización de nuestras áreas urbanas a través de políticas medioambientales transformadoras. Esta línea, junto con la identificación de nuevos espacios económicos vinculados a las comunidades locales (Casellas, 2010 y 2011), hubiera permitido iniciar un modelo urbano competitivo respecto a las economías emergentes, poco o nada sensibilizadas con cuestiones medioambientales y equitativas (Poli, 2010). Sin embargo, esta no ha sido la estrategia. En los dos siguientes apartados se analiza este aspecto. En primer lugar, se presentan las principales características de la restructuración capitalista en el periodo previo a la crisis. A continuación se identifican las políticas urbanas que han dominado hasta la fecha. La vinculación del proceso económico y las políticas urbanas nos permite ejemplificar el carácter reactivo de estas y, en consecuencia, su falta real de innovación.

\section{La reestructuración del capitalismo en el periodo previo a la crisis}

La restructuración del sistema capitalista iniciado en los años setenta como consecuencia de la crisis del petróleo provocó un cambio en ciudades de economías capitalistas avanzadas, las cuales iniciaron procesos de transformación de sus modelos productivos y su morfología urbana. Las características del modelo postfordista han sido ampliamente analizadas desde diferentes enfoques. Manuel Castells $(1997,1998)$ ha evaluado la relevancia de la revolución tecnológica y del conocimiento en la configuración de los nuevos espacios económicos y sociales; por su parte, David Harvey (1992) a través de un enfoque marxista ha aportado claves interpretativas a la reformulación del capitalismo y sus implicaciones sociales y territoriales. Desde el ámbito del análisis político y la teoría regulacionista, Bob Jessop $(1994,2002)$ ha explorado los nuevos modelos de gobernanza a diferentes escalas territoriales. Lash y Urry (1994) han aportado importantes reflexiones para entender los cambios culturales asociados al proceso de globalización y flexibilización de la economía. Dado 
su potencial analítico para el presente trabajo y como síntesis de las características de este proceso, en este artículo optamos por sumarnos a los factores identificados por Harvey (2010 y 2011).

Una de las estrategias de restructuración del sistema capitalista en las últimas décadas ha consistido en el aumento de la oferta de fuerza de trabajo a través de, o bien la deslocalización paulatina de empresas a países en vías de desarrollo o facilitar inmigración desde países más pobres a países de economías más avanzadas. Esta estrategia ha permitido que el capital tuviera acceso a una fuente extraordinaria de fuerza de trabajo a bajo coste, a la vez que paulatinamente ha deslegitimizado las reivindicaciones laborales del estado de bienestar occidental (Harvey, 2010). Esta transformación del estado de bienestar hacia un estado de carácter más emprendedor ha ido acompañada de un esfuerzo por priorizar las estrategias de desarrollo urbano de carácter económico, con un especial énfasis en la creación de infraestructura.

La importancia de la infraestructura como mecanismo incentivador de desarrollo económico se hace patente en el énfasis que instituciones como el Banco Mundial le otorgan. Por ejemplo, desde esta organización la infraestructura se sigue considerando una fuente básica para aumentar el crecimiento y lograr los Objetivos de Desarrollo del Milenio en países en vías de desarrollo y también como estrategia para reducir el desempleo en la Europa occidental. Esta prioridad ha implicado un claro detrimento de las políticas de carácter social, en la medida en que la promoción económica ha vehiculado las prioridades de las tradicionales políticas públicas (Hall y Hubbard, 1996; Casellas, 2007), a la vez que la magnitud de la inversión necesaria para financiar infraestructura ha implicado que a menudo los gobiernos locales representaran los intereses de los gobiernos centrales, los lobbies industriales y los mercados financieros internacionales en lugar de los intereses de los ciudadanos, que son quienes en última instancia los han elegido y a quien deben representar.

En lo relativo a la gobernanza, un factor clave que hay que considerar ha sido la progresiva pérdida de peso de los estados como instituciones reguladoras tradicionales, con una evolución hacia una posición de coordinación de intereses múltiples (Jessop, 2002). Ello ha repercutido, por un lado, en un aparente mayor papel ganado por las ciudades (Le Galès, 2003 y Jessop, 1994) y, por otro, en crecientes conflictos de redistribución entre diferentes niveles de gobernabilidad y grupos de interés (Peck y Tickell, 1994). Sin embargo, y a pesar de la narrativa dominante, que enfatiza la importancia de las ciudades, podemos argüir que las unidades superiores de gobernabilidad han desempeñado, y todavía desempeñan, un papel muy importante en cuanto a su influencia dentro de las características y los recursos con que cuentan las políticas urbanas. Por ejemplo, a escala regional y metropolitana, con una pluralidad de municipalidades de diferentes dimensiones, la provisión de infraestructura requiere la participación y financiación directa del estado. De este modo, el estado ha seguido manteniendo un importante control sobre la escala local, minimizando y mediatizando las decisiones políticas a escala urbana. Otro factor que debe considerarse, y que cuestiona la independencia de la escala local, involucra las 
características y afiliaciones de los candidatos en las elecciones municipales. Ello es evidente en el caso europeo, tanto en España como en Italia, donde la estructura de partidos con listas cerradas y controladas desde las organizaciones de los partidos políticos hace que los candidatos locales se encuentren estrechamente condicionados por los intereses de partido a escala supramunicipal.

Respecto a la actividad productiva, durante las décadas posteriores a la Segunda Guerra Mundial, los estados apoyaron las empresas nacionales establecidas en sus países de origen, principalmente a través de aranceles y otros mecanismos reguladores. Esta estrategia fue fundamental para que el estado de bienestar funcionara dentro del sistema capitalista. Al margen del marco de la guerra fría, que especialmente en Europa legitimaba los partidos socialdemócratas como vía intermedia entre el liberalismo anglosajón y el comunismo de los países del este, el capital aceptó las políticas sociales de carácter nacional porque generaban una demanda interna al expandir la clase media, a la vez que la producción se vendía ampliamente en los mercados nacionales protegidos, lo que creó un cierto monopolio para las empresas nacionales, que veían garantizadas sus plusvalías (Harvey, 2011). Este equilibrio empezó a alterarse a partir de los años ochenta, en parte respondiendo a las dinámicas de la globalización y bajo el auspicio de organizaciones como la Organización Mundial del Comercio. Este organismo desde los años noventa fomentó una estrategia de competencia interterritorial a escala global (Matsushita, et al. 2006), bajo criterios económicos neoliberales establecidos en lo que el economista Williamson (1990) denominó el Consenso de Washington. La propagación ideológica neoliberal se vio también beneficiada por la colaboración de institutos de investigación conservadores, como el American Enterprise Institute y el Institute of Economic Affairs. Sin embargo, a partir de finales de los años noventa, también se puso de manifiesto que esta estrategia de libre competencia y mercado, que a priori parecía beneficiar al capital industrial abriendo mercados, afectaba las dinámicas comerciales, tanto productivas como de consumo.

La competitividad a escala mundial generó a principios de la década del 2000 dos fenómenos contrapuestos. Por un lado, si bien el capital controlaba los costes laborales a través de la deslocalización y la inmigración ampliando sus mercados laborales, incluso en un contexto de congelación o disminución de salarios, los márgenes de beneficio sobre la producción industrial tradicional disminuyeron como consecuencia de la competencia global y continuada. Por otro lado, la introducción de nuevos procesos tecnológicos y la globalización generaron una superoferta de productos que las clases trabajadoras y medias de países occidentales no podían absorber debido al estancamiento de sus salarios. La respuesta a esta dicotomía, al margen de la expansión de la oferta hacía países emergentes con incipientes clases medias, como China, India o Brasil, fue la creación en los países occidentales de amplios nuevos mercados de consumo a través del acceso ilimitado al crédito y el segundo circuito del capital, en terminología de Lefevre (1991). En los siguientes apartados se analizan ambos fenómenos. 


\section{La ingeniería financiera}

La importancia del sector financiero como estrategia generadora de capital desde la década de los años noventa se alimentó a través de la ingeniería financiera. Tal y como Harvey (2010) apunta, la transformación profunda del sistema financiero se inició en 1986 con el llamado eufemísticamente Bing Bang, es decir, con la integración mundial de las bolsas y los mercados financieros para crear un único sistema global. A partir de este momento, aunque todavía persistían algunas barreras a la libre circulación de capitales, se permitía a nivel logístico y técnico la circulación de capital a escala global en busca de la mejor rentabilidad posible. Los bancos se internacionalizan y se eliminó la diferenciación entre bancos inversores y bancos de depósitos.

Con respecto al sector productivo, en el periodo anterior a la crisis, el alto nivel de beneficios del sistema financiero estimuló a empresas no financieras, como industrias, servicios a las personas y distribución, entre otras, a vehicular parte de su capital al sector financiero, ya que en él obtenían sus mejores beneficios. Un buen ejemplo de ello lo ofrece TESCO, la cadena de supermercados más importante de la Gran Bretaña, que incluso bajo la incertidumbre de una crisis financiera incipiente empezó a comercializar hipotecas en el 2008 con la colaboración del Royal Bank of Scotland (Worthington, 2008). La falta de mecanismos reguladores externos e internos al sistema financiero y bancario quedó ilustrada a través de la perversidad de los incentivos creados para altos cargos de las entidades. En el año 2008, Merrill Lynch perdió 27.000 millones de dólares; sin embargo, la empresa ofreció bonos por más de 1 millón de dólares a 700 empleados. Los cuatro primeros destinatarios recibieron un total de 121 millones de dólares (New York Times, 2009).

La creciente importancia de los diversos mecanismos financieros ideados para incentivar el crédito y la demanda, junto a las facilidades ofrecidas por los sistemas bancarios para financiar el consumo familiar y empresarial, actuó como mecanismo básico de la reestructuración económica, no solo para fomentar la producción/adquisición desproporcionada de productos, sino también en el proceso de urbanización del suelo. Como se ha apuntado, el incentivo crediticio se hacía necesario para mantener una demanda de consumo al alza en unas condiciones de falso crecimiento adquisitivo real para la población. De hecho, el poder adquisitivo de las familias y algunos sectores industriales supervivientes a la deslocalización habían estado estancados durante años, cuando no disminuidos en términos reales. Estudios realizados en los EUA demuestran que el consumo como porcentaje del PIB fue del $63 \%$ en 1980, del $67 \%$ en 1998 y del $70 \%$ en 2008 (Crotty, 2009). Productos financieros tóxicos, como el caso de las participaciones preferentes en España, son ejemplos de la falta de control y la opacidad de los productos bancarios, que en este caso captaron capitales de ahorradores no especulativos para refinanciar actividades de banca especulativa. De hecho, el carácter complejo de muchos de los productos financieros estructurados era tan elevado que eran inherentemente opacos incluso para inversores expertos. 


\section{El segundo circuito del capital}

En este marco de crecimiento económico, en la primera década del siglo XXI, la estrategia de desarrollo en economías avanzadas se contempló a través del segundo circuito del capital (Lefebvre, 1991), es decir, a través de la urbanización del suelo. Por un lado, el aumento del gasto de las familias fue impulsado por los efectos combinados del aumento de la deuda y, por otro lado, por la percepción de una mayor riqueza de los hogares creados por la burbuja inmobiliaria y bursátil. En EUA, mientras la deuda media de los hogares fue del 48 \% del PIB en 1985 — un dato similar al de 1965-, en 1998 había aumentado al $66 \%$ y a más del $100 \%$ a finales de 2008 (Crotty, 2009).

En el caso español, en el periodo 1998-2005 se construyó a un ritmo medio anual de casi 600.000 viviendas; dicho de forma más ilustrativa, se edificaron tantas unidades de vivienda como Alemania, Francia y la Gran Bretaña juntas. Las razones de este alto nivel de urbanización involucran tanto factores ideológicos como de inflación de la demanda y de la oferta (Burriel 2008 y 2011; Rodríguez, 2007). En el ámbito de la demanda, la inflación vino motivada por factores como: 1) los bajos tipos de interés, por debajo de la inflación; 2) los largos plazos de los préstamos-vivienda; 3) la participación de las entidades de crédito vía préstamos y participación directa en la promoción; 4) el rápido aumento del número de hogares fomentado por la inmigración y nuevos modelos familiares; 5) las compras de viviendas por extranjeros, y 6) la creciente presencia del inversor-especulador. Con relación a la oferta, cabe destacar: 1) el aumento continuado del precio del suelo; 2) la recalificación intensa de suelo como urbanizable residencial, y 3) el control de la propiedad del suelo por un número reducido de propietarios o grupos especulativos. Es remarcable que, a pesar del continuo crecimiento de suelo urbanizable, el coste del suelo subió en el periodo 1997-2006 a un ritmo equivalente al precio de la vivienda (Rodríguez, 2007). Un marco ideológico favorable a la desregularización y la deslegitimación del planeamiento favorecieron la burbuja (Burriel, 2008). La urbanización difusa característica de países anglosajones como los EUA se expandió en países como España, lo que aumentó los problemas de movilidad y la necesidad de inversión en nueva infraestructura y servicios.

\section{Las características y el impacto de las politicas urbanas reactivas}

En el análisis de la relación existente entre el proceso de globalización y los nuevos modelos de urbanización, importantes analistas han enfatizado la aparición de una nueva jerarquía urbana a escala mundial (Sassen, 2001) y han señalado la importancia ganada por las ciudades como nuevos centros de poder y decisión frente a otras escalas territoriales y organizativas como son los estados (Hall y Hubbard, 1996; Scott, 2009). También se ha destacado la creación de redes de ciudades con sus diversas zonas de influencia (Capello, 2000). Sin embargo, con respecto a la importancia de la escala local, a partir del análisis realizado en el apartado anterior se puede contraargumentar que, de hecho, el gobierno local ha aumentado su capacidad de actuación solo aparentemente, 
ya que en gran medida las municipalidades han seguido dependiendo de los recursos financieros aportados y diseñados desde la escala nacional o regional, especialmente en cuanto a infraestructura se refiere.

Con relación al creciente rol mediador del sector público, de hecho este podría interpretarse, desde una visión crítica, como la condición necesaria para seguir imponiendo criterios de arriba abajo en un periodo en el que la ciudadanía demanda participación activa en las decisiones urbanas. Desde esta óptica, la creciente importancia de la gobernanza urbana no respondería tanto a un proceso democrático real como a un aspecto formal necesario para conseguir cierto consenso y aprobación. De hecho, las amplias manifestaciones ciudadanas en ciudades españolas iniciadas a partir de la crisis, como el movimiento del 15-M bajo el lema Democracia real, ya, apuntarían a que la sociedad civil identifica la existencia de un modelo democrático formal, pero no real.

Existe consenso en considerar que el modelo capitalista no puede subsistir sin un constante proceso de crecimiento. Ello ha comportado que históricamente se hayan reproducido las crecientes tensiones entre, por un lado, la necesidad de mejorar la competitividad para garantizar la actividad empresarial y, por otro, la voluntad de mantener una cohesión social con políticas públicas en un contexto urbano cada vez más complejo (Fainstein, 2001). Desde esta perspectiva, se puede argumentar que la complejidad del proceso de globalización agudizó estas tensiones y ha influido en las características y prioridades de las políticas urbanas de las últimas décadas.

Desde los años noventa las empresas optaron crecientemente por la subcontratación o deslocalización del total de la producción hacia otros países con menores costes salariales y escasa regulación medioambiental. Paralelamente, la desinversión en el tejido industrial urbano y la creciente aparición de infraestructura obsoleta en ciudades occidentales con un pasado industrial fueron una constante a la que las políticas urbanas intentaron hacer frente. Para ello, los gestores urbanos, a menudo dentro de la cooperación público-privada, reinventaron las ciudades como centros de ocio y servicios avanzados en continuo proceso de readaptación. Estas políticas, lejos de mitigar las necesidades de inversión en infraestructura, las incentivaron, ya que la competitividad de los espacios urbanos se articuló a través de la necesidad de interconectividad a varias escalas territoriales y constante redefinición arquitectónica y urbanística para la creación de imagen de ciudad (Casellas, Dot, Pallares-Barbera, 2010).

En este esfuerzo por encontrar la diferenciación, se observó que las ciudades habían entrado en un proceso de competencia a escala internacional que empujaba a las economías locales a promocionarse no solo en el tipo de infraestructura urbana tradicional, «infraestructura fuerte» (hard infrastructure), sino también en la que se ha denominado «infraestructura suave» (soft infrastructure), en referencia a la calidad de servicios, como la educación, la sanidad, la cultura, el ocio, etc. Este tipo de infraestructura se convertía así en básica, en un intento por encontrar el elemento diferencial entre ciudades, cuando la globalización y la estandarización en los productos habían generado producción similar a escala global, tanto industrial como de consumo y cultural. 
La incentivación del turismo urbano, en concreto vinculándolo al turismo cultural, fue una de las estrategias complementarias orientadas al desarrollo de las economías locales. Desde principios de los años ochenta, las políticas de desarrollo europeo habían situado la cultura en el centro de las estrategias de regeneración urbana. El libro editado por Bianchini y Parkinson (1993) sobre políticas culturales en Europa identificaba casos de estudio de ciudades europeas punteras en esta estrategia. La utilización de la cultura como una estrategia de reconstrucción giraba en torno a la posibilidad de fomentar el desarrollo económico local, mientras que, de forma complementaria, se apoyaba las estrategias de marketing de la ciudad. En el caso de los Estados Unidos, las estrategias de desarrollo económico vinculado a la promoción cultural no se basaron en una política cultural integral, sino que se centraron en la creación de determinados centros culturales en zonas deprimidas que pudieran servir como incentivos para la inversión privada en la zona. Los nuevos proyectos culturales norteamericanos no solo se construyeron en los principales centros urbanos, tales como Nueva York o Chicago, sino también en ciudades con grandes dificultades sociales y económicas, como Newark, en Nueva Jersey, que durante décadas luchó contra la imagen negativa creada por los disturbios de mediados de los años setenta (Strom, 2002).

La cultura invertía así su significado histórico y pasaba de ser un subproducto de la riqueza a convertirse en generadora de riqueza. Sharon Zukin (1996) denominó este nuevo enfoque la economía simbólica. Una economía que se genera básicamente en el ámbito urbano y que se basa en la producción de símbolos como productos de consumo. La cultura genera directamente actividad económica, pero además fortalece la imagen de una ciudad al presentarla como innovadora y creativa. Así mismo, obliga a interrelacionarse con la oferta orientada al turismo cultural, lo que genera necesidad de coordinación entre políticas de la administración y las instituciones culturales locales, tanto del ámbito público como privado (Paül, 2013). En esta misma línea, las industrias del conocimiento ubican el diseño y la innovación en la vanguardia de su producción.

Dentro de este modelo de desarrollo local, la organización de un macroevento que propiciara una transformación urbana a gran escala se convirtió en otra estrategia perseguida globalmente por un amplio abanico de ciudades. Desde mediados de los años ochenta, tanto en ciudades europeas como norteamericanas, y a partir de los noventa a escala global, agentes políticos y económicos locales intentaron convertir sus ciudades en sedes de grandes acontecimientos deportivos, tales como juegos olímpicos o campeonatos de fútbol. Sin un gran debate en torno a la idoneidad de esta estrategia, los argumentos a favor de macroeventos se basaron en diversos criterios, incluyendo la transformación urbanística a gran escala, la atracción de inversión pública y privada, la creación de empleo y visibilidad y, por último, los amplios efectos multiplicadores indirectos e inducidos que un macroevento generaba dentro de la economía local. Un ejemplo excelente de este tipo de análisis lo ofrece el estudio de Lloyds Banking Group (2012) sobre el impacto económico de los Juegos Olímpicos de Londres de 2012. 
En los años noventa y en la primera década del siglo XXI, la especialización urbana en servicios avanzados y la cultura permitieron una cierta reaglomeración de actividad económica en ciudades occidentales. Sin embargo, como ya se ha apuntado, también se incrementó la dualidad social, ya que se convirtieron en centros de fuerza de trabajo especializado altamente remunerado y, simultáneamente, en polos de atracción para población nativa o inmigrantes de baja formación (Fainstein, 2001). En el caso de España, la presencia de fuerza de trabajo de baja cualificación se concentró en actividades de alto impacto medioambiental y urbanístico a través de la ejecución de programas de infraestructura física y construcción de vivienda que no respondían a necesidades reales, sino a la lógica de un crecimiento capitalista especulativo y a la inercia del sistema.

Paradójicamente, en el ámbito productivo, en el periodo de crisis iniciado en 2007, la presión sobre la necesidad de mantener puestos de trabajo permitió una mayor cooperación entre los intereses de las compañías y los sindicatos en la medida en que ambos presionaron de forma coordinada al sector público con la finalidad de mantener la actividad económica existente. Siguiendo las recomendaciones del Banco Mundial, los planes de estímulo a inicios de la crisis tuvieron un marcado carácter de inversión en urbanización e infraestructuras.

Históricamente, la inversión en infraestructura por parte del sector público ha sido una estrategia de inversión justificada, ya que durante décadas se la ha considerado una fuente generadora de riqueza, puestos de trabajo y desarrollo. De hecho, tradicionalmente, la mejora de la infraestructura ha sido condición sine qua non para el desarrollo económico. Sin embargo, en el contexto de la nueva economía y la presente crisis, se debería cuestionar de forma más amplia el beneficio marginal de la inversión pública que opta por ubicar recursos en un ámbito económico que produce puestos de trabajo no cualificados y graves costes medioambientales e incrementa la necesidad de energía y movilidad inducida, lo que repercute negativamente en la calidad de vida de los ciudadanos. La inversión en infraestructura física debería compararse con las inversiones en actividades de nueva economía y culturales, que no solamente tienen un impacto medioambiental más bajo y emplean un mayor número de trabajadores cualificados, sino que también ofrecen la posibilidad de que su producción total sea más rentable y más efectiva en la mejora de la calidad de vida de los ciudadanos. El esfuerzo por encontrar vías alternativas y cuestionar la idoneidad de la inversión en infraestructura, en este caso de transporte, se aborda en la siguiente sección.

\section{La movilidad a partir de la innovación política y social}

En el anterior apartado se ha identificado aspectos fundamentales de la evolución económica de las últimas décadas y su repercusión en las políticas de restructuración urbana. Como el análisis permite vislumbrar, se puede argumentar que las políticas adoptadas han sido a menudo reactivas más que proactivas. Las ciudades han intentado solventar problemas y adaptarse a la racionalidad 
aportada por los mercados. Así mismo, los procesos innovadores de carácter social, político y organizativo han tenido escasa repercusión. La inversión se ha realizado a partir de esquemas tradicionales y con un amplio coste para el sector público, con resultados a menudo muy cuestionables.

En cuanto a infraestructuras de transporte, en España la estrategia en movilidad se ha basado en la ampliación de infraestructura a todos los niveles, dentro de la particularidad de primar los criterios de estado con centralidad en Madrid por encima de los criterios de eficiencia económica. El análisis realizado por Germà Bel (2011) acerca de las características de las infraestructuras españolas, tanto en el ámbito aeroportuario, como vial y de red ferroviaria, permite observar el proceso histórico de creación de estado. Ello explicaría la reiterada voluntad centralizadora de las infraestructuras, que con un marcado carácter radial con centralidad en Madrid, se han ejecutado reiteradamente al margen de su efectividad económica. A escala más local, la movilidad ha seguido propiciando la creación de mercados laborales metropolitanos, con el consiguiente mecanismo de movilidad inducida y baja efectividad en la mitigación de los problemas del tráfico.

En este contexto, la crisis estructural del sistema capitalista iniciada a partir del 2007 ha generado una creciente restricción de los recursos, tanto públicos como privados, lo que ha agudizado la necesidad de replantear las estrategias territoriales. De hecho, se puede argumentar que el problema de la movilidad urbana resulta ilustrativo del presente fracaso de las políticas urbanas en general. Si consideramos el tráfico como síntoma, podemos argumentar que el tráfico caótico, caro y contaminante de nuestros espacios urbanos es el síntoma de una problemática mucho más grave que el reto de mover de un lugar a otro millones de personas cada día. Por este motivo, el tema del tráfico puede abordarse como un caso específico extrapolable a otras políticas urbanas.

El tráfico y los transportes son una cuestión crucial cuando se desea abordar el tema de la innovación urbana contemporánea, ya que la ciudad moderna se organiza alrededor de la movilidad de masas. Las ciudades contemporáneas han pasado de ser lugares en los que se vivía a ser áreas a donde se viaja por razones de trabajo, de ocio o de acceso a servicios (Poli, 2009). Aplicando un nuevo enfoque de análisis a partir de la consideración de la innovación como una cuestión social y política, y tomando en consideración las presentes limitaciones en movilidad, podemos argumentar que las barreras estructurales que impiden el cambio, y por ello una innovación respecto a la presente situación, en el caso específico de la movilidad se pueden identificar como: 1) la presente estructura del gasto público, 2) el poder de presión de las empresas que actúan en el sector de los transportes y la creación de infraestructura, 3) la formación de los especialistas en la planificación del tráfico y del transporte, y 4) el desconocimiento de las preferencias de los consumidores y, por tanto, la dificultad para comunicar las nuevas conductas y preferencias y, en consecuencia, la aplicación de posibles soluciones novedosas (Poli, 2011).

Ante esta problemática, y centrándonos en la cuestión del tráfico, se pueden proponer tres acciones innovadoras que tienen un potencial de influencia en 
la presente estructura urbana. La primera acción hace referencia a la inclusión de la participación efectiva de los ciudadanos en el proceso de identificación de necesidades; la segunda involucra el uso del potencial que ofrecen las nuevas tecnologías; finalmente, la tercera implica la exploración de instrumentos fiscales y económicos novedosos.

La inclusión de la participación efectiva de los ciudadanos en el proceso de identificar preferencias requiere un replanteamiento de los términos de participación ciudadana. Este replanteamiento conlleva acabar con la extendida práctica de informar de planes y recoger sugerencias. La inclusión participativa fructífera requiere mecanismos eficientes para identificar las conductas y las preferencias de los ciudadanos. Por ello, la información debe ser pública y transparente y debe estar orientada a investigar nuevas vías de comunicación que incluyan la identificación de los mecanismos de cambio de los comportamientos de los individuos, el diseño de nuevos posibles escenarios urbanos y la legitimación de los procesos democráticos y de decisión.

Con relación al diseño de soluciones, para incluir el potencial que ofrecen las nuevas tecnologías de forma creativa, se debe abrir el marco de análisis para explorar el potencial del trabajo a distancia, un nuevo modelo de logística y un uso generalizado de las telecomunicaciones y de la información. Finalmente, la aplicación de instrumentos fiscales y económicos debe comportar la aprobación de nuevas normativas que creen la posibilidad de introducir nuevas soluciones espaciales, como por ejemplo aprobar leyes para la conservación de espacios verdes y áreas diversificadas en vez de los usos específicos del suelo.

Así mismo, la reducción de la movilidad diaria se podría basar en una política residencial flexible que favorezca la cercanía entre los puestos de trabajo y la residencia, en vez de favorecer las llamadas áreas de mercado laboral o áreas metropolitanas laborales. Desde hace más de sesenta años, en Europa se ha intentado resolver el problema del tráfico aumentando la movilidad. En el presente contexto se deberían promover investigaciones para reducir la movilidad respondiendo a preguntas como la siguiente: ¿es más eficaz construir una autopista que aumente la oferta de movilidad un $5 \%$ o invertir la misma cantidad de recursos materiales en residencias y puestos de trabajo que reduzcan dicha movilidad? Esta impostación del problema de la movilidad implica una investigación y una tecnología completamente diferentes de las actuales.

El mismo reto intelectual se puede trasladar a la planificación urbana de calles y plazas. En este campo ya se ha innovado en Europa y se han ido introduciendo cambios significativos. Pero el paso siguiente y más radical de la innovación sería crear espacios urbanos utilizables por aquella parte de la ciudadanía que demanda menos movilidad. En la actualidad, gran parte de las investigaciones y de las innovaciones, y consecuentemente de la financiación, está concentrada en la demanda de movilidad. Se argumenta que esto sucede porque hay un mercado, pero de hecho la relación de causalidad se puede argumentar a la inversa. Se puede pensar que hay un mercado porque hay una oferta, mientras que, en el presente, para aquellas las personas que reivindican estilos de vida diferentes no hay un mercado o un modelo que responda a sus necesidades. 
Otro sector de investigación e innovación para el que no existe una demanda real, pero sí potencial, concierne a todos los usos secundarios del automóvil que podrían ser substituidos por otra clase de intervenciones. Es fácil para los ambientalistas luchar contra el número excesivo de automóviles en las ciudades. Sin embargo, también debemos observar que los coches no contaminan solo cuando se mueven, sino también cuando están estacionados, ya que ocupan valiosos espacios urbanos que podrían dedicarse a otros usos. De hecho, la utilización que damos a los coches estacionados puede ser cubierta por otras soluciones. Habitualmente utilizamos el coche como depósito de equipajes, paraguas, lugar de encuentros, para descansar, etc. Algunos de estos usos pueden substituirse con soluciones variadas. Si se planifican los espacios teniendo en cuenta los usos secundarios e impropios de los coches, se puede reducir la ocupación de suelo urbano.

Siguiendo esta misma línea argumental, nos podemos cuestionar la escala en la que organizar el transporte público o, mejor dicho, colectivo. Se pueden invertir más o menos fondos para el transporte de larga distancia o de cercanías. Por ejemplo, hay todavía una diferencia desproporcionada entre las inversiones para el transporte en áreas de alrededor de diez hectáreas respecto al transporte regional. Esta es una elección más ideológica que técnica. La misma carencia se encuentra en lo que concierne a las políticas de distribución de la venta al detalle. La compra de proximidad es una opción que satisface a los comerciantes tradicionales, normalmente arraigados económica y socialmente a barrios. Proyectos para favorecer la compra de proximidad cuentan con un gran apoyo del pequeño y mediano comercio y un creciente número de ciudadanos.

Otra posibilidad responde a la innovación en la búsqueda de mercados de trabajo local en el mismo barrio de residencia. Un buen ejemplo de dinámicas laborales y residenciales lo ofrecen los campus universitarios, especialmente en los países anglosajones, los cuales ofrecen a los estudiantes trabajos a tiempo parcial en las bibliotecas, cafeterías, secretarías, etc. Ello es posible porque las universidades son comunidades cerradas donde la información es amplia y abierta y no está burocratizada. La información se encuentra a disposición ya sea como oferta o demanda de trabajo. Esto nos permite tener servicios que no podrían ser satisfechos de forma tan eficiente a una mayor escala.

El trabajo a distancia y la telemática en general favorecen una reducción de la movilidad física, pero también una organización diferente de la ciudad en su conjunto. Hace más de veinte años que nuevos proyectos fueron puestos en marcha en Estados Unidos y también en Europa. El problema es que se pretenden resultados inmediatos en la estructura urbana y en el estilo de vida. Sin embargo, estos cambios son lentos; de hecho, tan lentos como los cambios que se producen cuando se construye una nueva infraestructura física. La diferencia es que para el trabajo a distancia y otros cambios descritos se pretende obtener resultados inmediatos.

Una mejor información a escala local también permitiría utilizar servicios de cercanía en vez de disponer de una vasta área en donde buscarlos. En este caso se trataría de cambiar la orientación de nuestras investigaciones, lo que 
requiere un esfuerzo creativo e intelectual que abra nuevos caminos en lugar de seguir los ya conocidos. Finalmente, el dualismo entre el transporte privado y el público tiene que ser discutido en mayor profundidad. Hay proyectos y soluciones todavía poco aprovechados basados en tecnologías que garantizarían el aprovechamiento de un vehículo y otras formas de compartir los medios de transporte privados. Si tenemos en cuenta los asientos vacíos en los coches particulares que cada mañana observamos en el tráfico urbano, podemos darnos cuenta del derroche de recursos y de la inutilidad de una gran parte del transporte público, que podría ser sustituido por una organización diferente en la que el transporte individual actúe como transporte público.

Para realizar este nuevo enfoque es necesario poner en marcha una nueva formación de los gestores urbanos y poner en valor competencias diferentes e innovadoras. Esto implica una política de reforma de los colegios profesionales y de la educación. Pero sobre todo se requiere una política capaz de promover el cambio y de no estar condicionada por los intereses de grupos económicos o profesionales consolidados.

Cabe enfatizar que una pieza clave para el cambio de paradigma es la introducción de cambios en la formación académica de los expertos en movilidad. Para ello se requieren nuevos programas de formación universitaria que eduquen a los profesionales para tratar el tráfico de manera más social y humanística, concentrándose en los aspectos sociales y medioambientales. Como consecuencia de este replanteamiento, la innovación en temas de movilidad se debería plantear como el fruto de investigaciones desde disciplinas complementarias y con objetivos diversos.

\section{Reflexiones finales}

La palabra dinámica proviene de la palabra griega dynamos, que significa fuerza. En este sentido, cuando nos referimos a la dinámica en la organización del espacio urbano podríamos hablar de una fuerza espontánea, natural y sistémica, casi en contraste con una acción humana libremente elaborada y realizada conscientemente. La dinámica —es decir, un proceso que tiene lugar de manera espontánea respondiendo a fuerzas naturales - es lo opuesto a la planificación, la cual implica una serie de elecciones racionales o por lo menos conscientes. Sin embargo, si asociamos dinámica a innovación reconocemos la posibilidad de intervenir en el proceso, porque el concepto de innovación contiene entonces en sí mismo la idea de cambio.

En esta nueva exploración de interrelación entre innovación y dinámicas espaciales, si seguimos entendiendo la innovación solo como una cuestión técnica, no avanzamos en el proceso epistemológico. Si, por el contrario, la innovación incluye nuevos procesos educativos, una nueva definición de objetivos y de cambios en la política urbana, en la economía y en la tecnología, entonces la innovación vuelve a formar parte del pensamiento racional y genera la posibilidad de actuar a partir de decisiones tomadas desde el ámbito de la libertad y la política. 
Con este enfoque, la innovación se desplaza de la esfera técnica para situarse en el ámbito social, ético y de la organización de la producción y del consumo. Si queremos cambiar una tendencia consolidada, entonces debemos centrarnos en cambiar estructuras económicas y políticas. Como consecuencia de ello, la temática de la innovación debe considerarse básicamente un ámbito político; de lo contrario, podemos continuar hablando de una innovación que no cambia ni cambiará absolutamente nada. La innovación no tiene por qué estar incluida en las dinámicas territoriales como si fuese una consecuencia de ellas mismas; sin embargo, la innovación, pensada y elegida libremente, tiene que determinar las dinámicas territoriales, que a su vez tenemos que querer y elegir libremente.

A pesar de que podamos considerar que las ciudades se adaptan rápidamente a los cambios propiciados por la tecnología, de hecho los cambios se producen muy lentamente. Así, aunque en las últimas décadas, de la mano de la urbanización dispersa, las ciudades europeas se han acercado más al modelo de las ciudades americanas, bajo muchos puntos de vista nuestras ciudades han respondido desde hace poco, y no completamente, a la tecnología de la motorización de masa. Las estrategias que las ciudades occidentales han adoptado en el periodo previo a la crisis han implicado cambios respecto a décadas anteriores; sin embargo, uno de los elementos más significativos en políticas urbanas ha sido la proliferación de estrategias similares, independientemente de las características específicas de las ciudades.

En la presente situación de crisis, el problema de la innovación, la planificación y la dinámica territorial tiene que ver de forma incisiva no tanto con las cuestiones meramente técnicas sino con aspectos políticos y sociales; por ello, la innovación debería estar estrechamente relacionada con la política de la ciencia y de la técnica, al igual que con los mecanismos de poder que los colegios profesionales y los modelos educativos e industriales vehiculan a la sociedad. Como consecuencia de ello, el rol político de la educación universitaria se vuelve, una vez más, decisivo.

\section{Bibliografía}

BECK, U. (1992). Risk society: towards a new modernity. Londres: Sage.

BEL, G. (2011). Espanya, capital París. Barcelona: La Campana.

BIANCHINI, F. y PARKINSON, M. eds. (1993). Cultural policy and urban regeneration: the West European experience. Manchester: Manchester University Press.

BURRIEL, E. (2008). «La "década prodigiosa" del urbanismo español (1997-2006)». Scripta Nova. Revista Electrónica de Geografia y Ciencias Sociales, XII (270). <http:// www.ub.edu/geocrit/sn/sn-270/sn-270-64.htm>.

- (2011). «Subversion of land-use plans and the housing bubble in Spain». Urban Research \& Practice, 4 (3), 232-49.

CAPELlo, R. (2000). «The city network paradigm: measuring urban networks externalities». Urban Studies, 37 (11), 1925-45.

CASELLAS, A. (2007). «Gobernabilidad, participación ciudadana y desarrollo económico: adaptaciones locales a estrategias globales». Scripta Nova. Revista Electrónica de 
Geografía y Ciencias sociales. Barcelona: Universidad de Barcelona, XI (243) (10 de julio de 2007) <http://www.ub.es/geocrit/sn/sn-243.htm>.

- (2010). "La geografía crítica y el discurso de la sostenibilidad. Perspectivas y acciones». Documents d'Anàlisi Geogràfica, 56 (3), 573-81.

- (2011). «La crisis, la geografía económica y Julie Graham: alternativas de desarrollo local a partir de la crítica feminista». Anales de Geografía de la Universidad Complutense, 31 (2), 31-46.

Casellas, A.; Dot, E. y Pallares-Barbera, M. (2010). «Creación de imagen, visibilidad y turismo como estrategias de crecimiento económico de la ciudad». Finisterra XIV, 90, 153-72.

CASTELls, M. (1997). The power of identity: The information-age economy, society and culture. Malden, MA: Blackwell Publishers.

- (1998). End of millennium. Oxford: Basil Blackwell.

CROTTY, J. (2009). «Structural causes of the global financial crisis: a critical assessment of the new financial architecture». Cambridge Journal of Economics, 33 (4), 563-80.

FAINSTEIN, S. (2001). "Competitiveness, cohesion and governance: their implications for social justice». International Journal of Urban and Regional Research, 25 (4), 884-8.

Hall, T. y Hubbard, P. (1996). "The entrepreneurial city: new urban politics, new urban geographies?». Progress in Human Geography, 20 (2), 153-74.

HaRVEY, D. (1992). The condition of postmodernity. An enquiry into the origins of cultural change. Chichester: John Wiley \& Sons.

- (2010). The enigma of capital and the crises of capitalism. Londres: Profile Books.

- (2011). "Crisis, geographic disruptions and the uneven development of political responses». Economic Geography, 87 (1), 1-22.

LuHMANn, N. (1990). Sociedad y sistema: la ambición de la teoría. Barcelona: Pairós.

Jessop, B. (1994). «Post-fordism and the state». En: Amin, Ash (ed.) Post-fordism: a reader. Cambridge: Blackwell, 251-79.

- (1995). «The regulation approach, governance, and post-fordism: alternative perspectives on economic and political change?». Economy and Society, 24 (3), 307-33.

- (2002). «Liberalism, neoliberalism and urban governance: A state-theoretical perspective». Antipode, 34 (3), 452-72.

LASH, S. y URRY, J. (1994). Economies of signs and space. Londres: Sage.

LE GALES, P. (2003). Le retour des villes européennes: sociétés urbaines, mondialisation, gouvernement et gouvernance. París: Presses de Sciences Po.

LeFEBVRE, H. (1991). The production of space. Oxford: Blackwell.

LLOYDS BANKING GROUP (2012). London 2012: what is the economic impact on the UK? <http://www.lloydsbankinggroup.com/media/pdfs/lbg/2012/Eco_impact_report. pdf> [10 de noviembre de 2012]

LuHMANn, N. (1990). Sociedad y sistema: la ambición de la teoría. Barcelona: Paidós Ibérica.

Matsushita, M.; Schoenbaum, T. y Mavroidis, P. C. (2006). The World Trade Organization. Law, practice and policy. Oxford: Oxford University Press.

New YORK Times (2009). "After losses, a move to reclaim executives' pay». 22 de febrero de 2009.

PAÜL I Agustí, D. (2013). «Las políticas culturales y sus repercusiones en la imagen de la ciudad». Scripta Nova. Revista Electrónica de Geografía y Ciencias Sociales. [En línea]. Barcelona: Universidad de Barcelona, XVII (432). <http://www.ub.es/ geocrit/sn/sn-432.htm>.

PeCK, Jamie y Tickell, A. (1994). "Searching for a new institutional fix». En: Amin, Ash (ed.). Post-fordism: a reader. Cambridge: Blackwell, 280-315. 
POLI, C. (2009). Città flessibili. Una rivoluzione nel governo urbano. Turín: Instar Libri.

- (2010). "Sustainable development: from fallacy to fraud». Human Geography. A New Radical Journal, 3 (2), 63-82.

- (2011). Mobility and environment. Humanists versus engineers in urban policy and professional education. Londres: Springer.

RodrígUEZ, J. (2007). "Urbanismo, vivienda y economía en España». Arquitectura, Ciudad y Entorno, 1 (3), 80-85.

Sassen, S. (2001). Global cities: New York, London, Tokyo. Princeton, NJ: Princeton University Press.

SCOTт, A. J. (2009). Social economy of the metropolis: cognitive-cultural capitalism and the global resurgence of cities. Oxford: Oxford University Press.

STROM, E. (2002). «Converting pork into porcelain: cultural institutions and downtown development». Urban Affairs Review, 38 (1), 3-21.

Williamson, J. (ed.) (1990). Latin American adjustment: how much has happened? Washington: Institute for International Economics.

WORLD BANK (1991). Urban policy and economic development: an agenda for the 1990s. Washington DC: World Bank Publications.

WORTHINGTON, S. (2008). «Retailers' entry into the provision of financial services: the case for and against». International Journal of Financial Services Management, $3(3-4), 243-354$.

Zukin, S. (1996). The cultures of cities. Londres: Blackwell Publishers. 